\section{Testergebnis, richtig-negatives}

R.-D. Hilgers ${ }^{1}$, N. Heussen ${ }^{1}$ und S. Stanzel ${ }^{2}$

${ }^{1}$ Institut für Medizinische Statistik, Universitätsklinikum der RWTH Aachen, Aachen, Deutschland

${ }^{2}$ Heidelberg, Deutschland

Synonym(e) Richtig-negativer Test

Englischer Begriff true-negative test; true-negative test result
Definition Ein richtig-negatives Ergebnis eines diagnostischen Tests ( $\triangleright$ Test, diagnostischer) liegt vor, wenn tatsächlich Gesunde ein negatives Testergebnis aufweisen.

Beschreibung Richtig-negative Testergebnisse beschreiben die korrekten Testergebnisse für das Nichtvorliegen der Krankheit. Die Anzahl richtig-negativer Testergebnisse wird durch den Wert $d$ geschätzt (Bezeichnungen s. Tabelle im Stichwort $>$ Vierfeldertafel).

\section{Literatur}

Hilgers R-D, Bauer P, Scheiber V (2002) Einführung in die Medizinische Statistik. Springer, Berlin/Heidelberg/New York 